\title{
Research Paper \\ The Relationship Between Sexual Performance and Attitude Toward Menopause in Postmenopausal Women Referred to Health Centers Of Torbat Heydariyeh in 2017
}

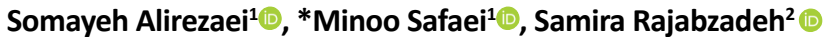

1. PhD. Student of Reproductive Health, Student Research Committee, School of Nursing and Midwifery, Mashhad University of Medical Sciences, Mashhad, Iran 2. Department of Midwifery, Faculty of Nursing and Midwifery, Torbat Heydariyeh University of Medical Sciences, Torbat Heydariyeh, Iran.

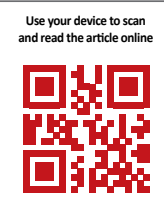

Citation: Alirezaei S, Safaei M, Rajabzadeh S. [The Relationship Between Sexual Performance and Attitude Toward Menopause in Postmenopausal Women Referred to Health Centers Of Torbat Heydariyeh in 2017 (Persian)]. Iranian Journal of Ageing. 2020; 14(4):510-519. https://doi.org/10.32598/sija.13.10.270

doi) https://doi.org/10.32598/sija.13.10.270
Key words:

Attitude, Menopause, Sexual function

\section{A B STRACT}

Received: 25 May 2018 Accepted: 15 Dec 2018 Available Online: 01 Jan 2020

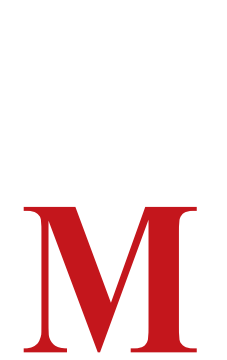

\section{Extended Abstract}

\section{Introduction}

enopause is a fundamental change in a woman's life with various essential aspects. Besides, attitudes toward it affect the management of this period [1]. Despite the similarity of biological events in menopause, attitudes toward menopause vary between different cultures and societies [2]. Such differences, in addition to menopause, affect sexual function during this period [3]. Menopause could be influential in causing, aggravating, or eliminating sexual complications. According to reports, $35 \%$ of postmenopausal women suffer from decreased sexual desire [4]. Iran is a multicultural country where certain sociocultural factors affect sexual function [5]; however, studies in this field are scarce [6]. Therefore,

\section{* Corresponding Author:}

\section{Minoo Safaei, PhD. Candidate}

Address: Student Research Committee, School of Nursing and Midwifery, Mashhad University of Medical Sciences, Mashhad, Iran.

Tel: +98 (915) 6595961

E-mail: safaeim1@gmail.com 
the current study aimed to investigate the relationship between sexual function and attitude toward menopause in postmenopausal women.

\section{Methods \& Materials}

The present descriptive study was conducted on 200 postmenopausal women referring to health centers in Torbat-e Heydarieh City, Iran, in 2016. By cluster and convenience sampling methods, healthcare centers and menopausal women were selected, respectively. The study inclusion criteria included Iranian citizenship and having sexual relationships with a spouse. Moreover, the study exclusion criteria were abnormal and premature menopause, diabetes, cardiovascular disease, cancer, as well as malformations or injuries of the genital area. We conducted the present research after receiving the approval of the Ethics Committee and obtaining written consent forms from the study samples.

Research tools included demographic data form, the Menopause Attitude Scale (MAS), and Female Sexual Function Index (FSFI). The MAS includes 20 items and 5 different dimensions; reasoning, goals, achieving aspirations and goals, self-concept, and appropriateness. This questionnaire is answered by a two-answer scale, including yes and no; a score of 0 is dedicated to disagreeing and 1 for agreeing. Its average obtainable score equals 12.4 . The FSFI consists of 19 questions that measure 6 dimensions of sexual function (desire, arousal, lubrication, orgasm, satisfaction, \& pain during intercourse) in the past 4 weeks. The appropriate cut-off point for sexual function detection was considered as 26.55 . The validity of the MAS and sexual function questionnaire was evaluated implementing a content validity approach. The reliability of the sexual function and MAS was determined by the internal consistency method and calculating Cronbach's alpha coefficient $(\mathrm{r}=0.82$ \& $r=0.78$, respectively). After sampling, the obtained data were analyzed using SPSS and statistical tests. $\mathrm{P}<0.05$ was considered significant.

\section{Results}

A total of 200 postmenopausal women aged 85-45 years were investigated in this study. The Mean \pm SD age of the explored women was $7.93 \pm 57.18$ years. The highest age distribution fell in the range of 51 to 55 years $(53.7 \%)$. The Mean \pm SD duration of menopause was $7.00 \pm 7.00$ years in the study participants. Furthermore, 176 (89\%) females were housewives and $167(83.5 \%)$ were educated below high-school diploma. The income of 145 (72.5\%) study samples were reported to be sufficient. The FSFI scores revealed that the mean total score of sexual function was equal to $22.53 \pm 5.91$ (Range: 14.40-35.60). Table 1 presents 6 areas of sexual function in percentage.

The present research results indicated that the lowest obtained score belonged to the area of sexual desire $(2.96 \pm 1.11)$ and the highest score was related to the area of sexual satisfaction $(4.40 \pm 1.05)$. The scores of the other domains were as follows: sexual arousal (3.25 \pm 1.35$)$, lubrication (4.03 \pm 1.29$)$, orgasm $(3.85 \pm 1.26)$, and pain (4.02 \pm 1.36$)$. In total, $27.5 \%$ of all studied postmenopausal women presented desirable sexual performance and $35.5 \%$ of them had a positive attitude toward menopause.

The Chi-squared test data revealed that the postmenopausal women (45.5\%) who had an undesirable sexual function, also reported a negative attitude towards menopause; only $8.5 \%$ of these women had both desirable sexual performance and a positive attitude towards their menopause. However, the Chi-squared test results suggested no significant relationship between sexual function and attitude toward menopause (Table 1). In other words, the sexual function did not affect attitude toward menopause $(\mathrm{P}=0.07)$.

Table 1. The relationship between sexual function and attitude toward menopause in postmenopausal females*

\begin{tabular}{|c|c|c|c|c|}
\hline \multirow{3}{*}{ Sexual Function } & \multicolumn{3}{|c|}{ No. (\%) } & \multirow{3}{*}{$\mathbf{P}$} \\
\hline & \multicolumn{2}{|c|}{ Attitude Toward Menopause } & \multirow{2}{*}{ Total } & \\
\hline & Negative & Positive & & \\
\hline Desirable & $38(19)$ & $17(8.5)$ & $55(27.5)$ & \\
\hline Undesirable & $91(45.5)$ & $54(27)$ & $145(72.5)$ & 0.07 \\
\hline Total & $129(64.5)$ & $71(35.5)$ & $200(100)$ & \\
\hline
\end{tabular}

${ }^{*}$ Chi-squared test 


\section{Discussion}

The present study demonstrated no relationship between sexual function and attitude toward menopause in the study participants. The sexual function of postmenopausal women did not affect their attitudes toward menopause. In total, $64 \%$ of the explored women presented a negative attitude toward menopause. Menopause is among the most critical stages of a woman's life and is associated with numerous symptoms and complications, including sexual dysfunction; thus, having sufficient and positive awareness and attitude about this phenomenon is a logical manner to cope with it [7].

Accordingly, optimal planning is required to modify women's attitudes and improve their health status and quality of life. A limitation of this study was the restricted range of samples in terms of demographic characteristics, and generalizability of the obtained results. It is suggested that future research provides training on menopause and sexual function.

\section{Ethical Considerations}

\section{Compliance with ethical guidelines}

We conducted the present research after receiving the approval of the Ethics Committee|: IR.THUMS.REC1397.29 and obtaining written consent forms from the study samples.

\section{Funding}

The present study is the result of a research project with the code IR.THUMS.REC.1395.5 of Torbat Heydariyeh University of Medical Sciences, which was supported with the financial support of the Vice Chancellor for Research.

\section{Authors' contributions}

Conceptualization, methology, software, validation, supervision, project administration: Minoo Safaei; Formal analysis, resources, data curation, writing-original draft preparation, writing review \& editing, visualization: Somayeh Alirezaei; Investigation: Samira Rajabzadeh; Funding acquisition: All authors.

\section{Conflicts of interest}

Authors declare no actual or potential conflict of interest related to this study.

\section{Acknowledgements}

We thank of the Torbat Haydariyeh University of Medical Sciences to provide the resources needed for this report. 


\title{
بررسى رابطه بين نكَرش نسبت به يائسكَى و عملكرد جنسى در زنان يائسه مر اجعه كننده به مراكز بهداشتى -درمانى شهرستان تربت حيدريت يدريه
}

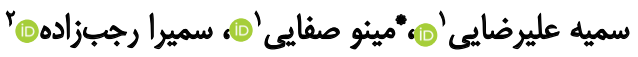 \\ ا. دانشجوى دكترى بهداشت بارورى، كميته تحقيقات دانشجويى، دانشكده يرستارى و مامايى، دانشُكاه علوم يزشكى مشهيده مشهده ايران.

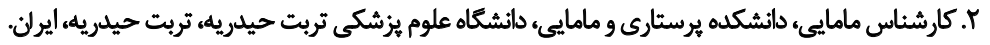

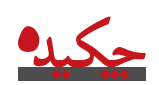

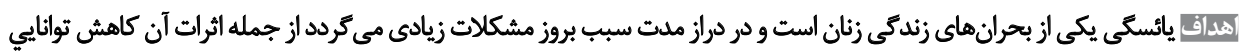

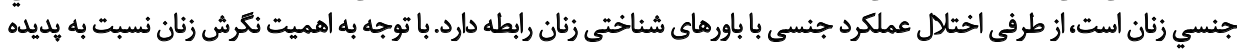

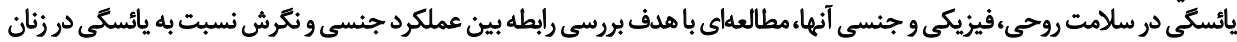

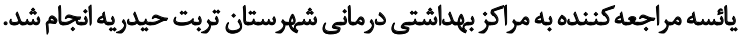

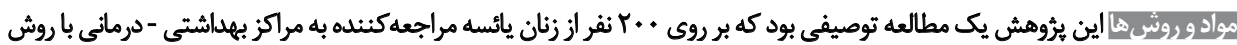

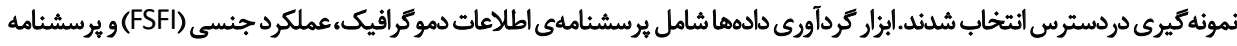

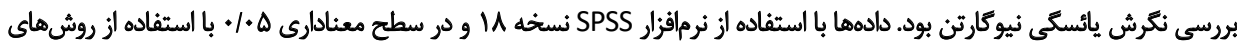

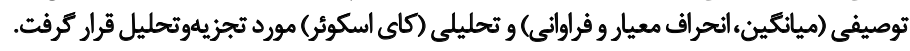

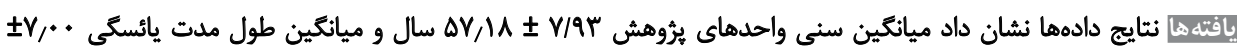

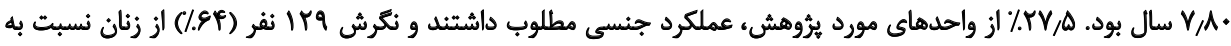

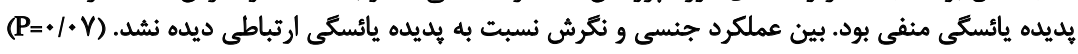

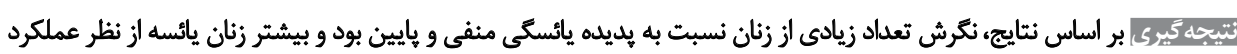

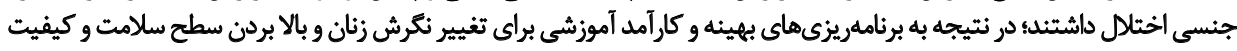
زندكى ايشان نياز است.

تاريخ دريافت: F+ تير Ta

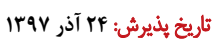

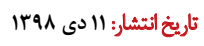

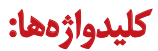

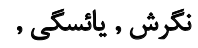

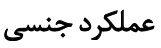

درد مفاصل، تحريكيذيرى و اضطراب، احساس غم، افسردگى و تعريق شبانه رنج مى برند [برئ.

dolê.

با توجه به اينكه يائسكى يك تغيير اساسى در زندكى يك

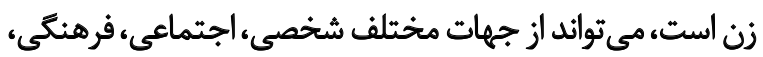

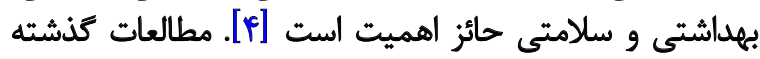

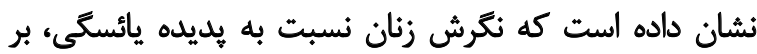

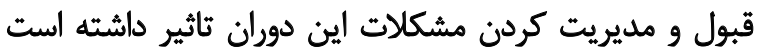

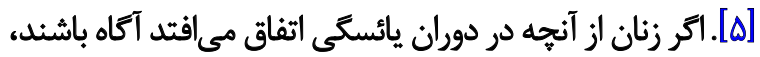

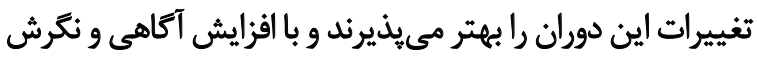

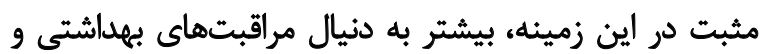

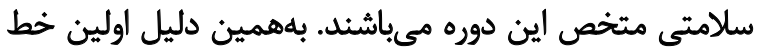

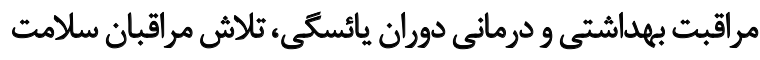

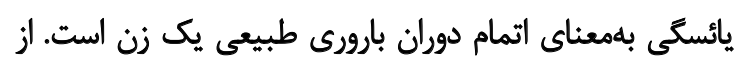

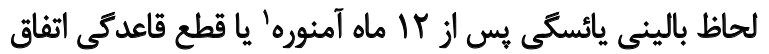

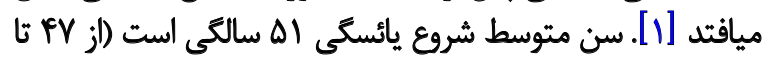

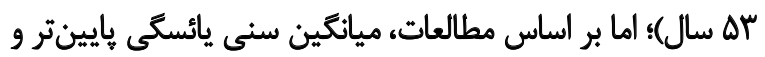

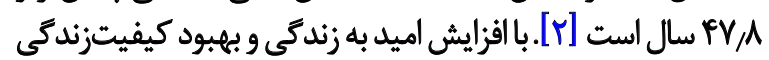

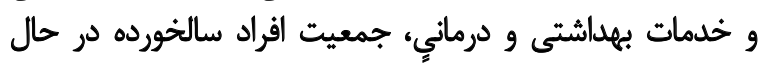

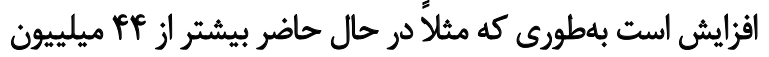

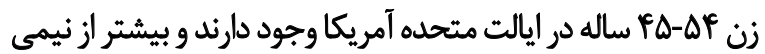

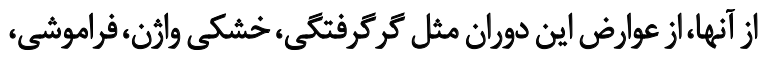

\section{Amenorrhea}

* نويسنده مسئول:

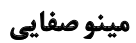

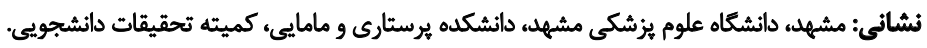
تلفن: يست الكترونيكي: safaeim1@gmail.com 
طبق كزارشات هـ/ از زنان يائسه از كاهش ميل جنسى رنج

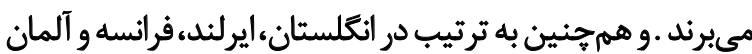

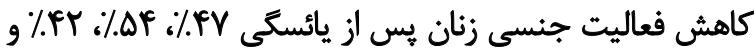

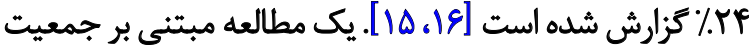

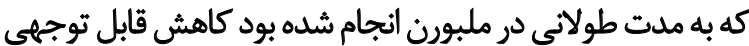

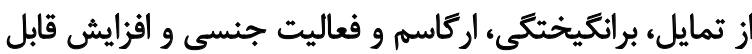

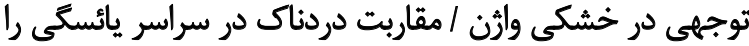

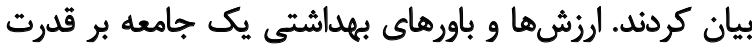

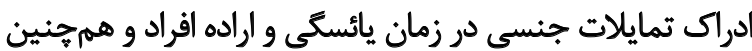

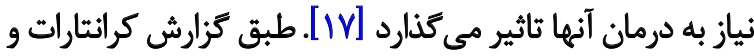

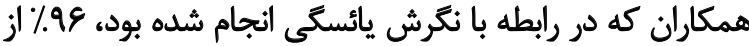

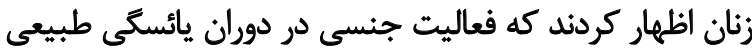

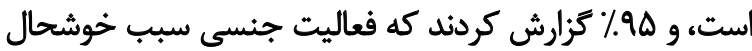

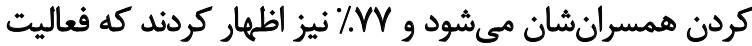

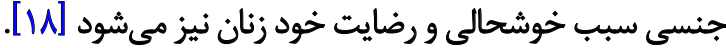
در بررسى بين مليتهاى مختلف، زنان يائسه سياه يوست و وائري

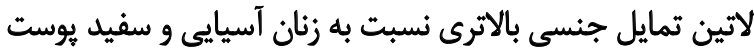

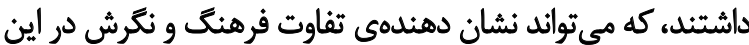

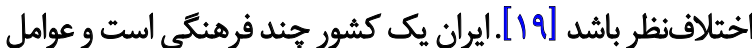

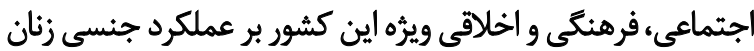

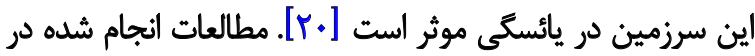

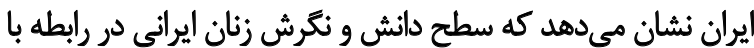

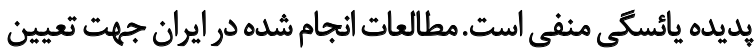

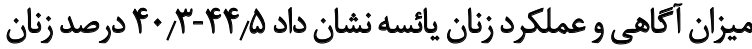

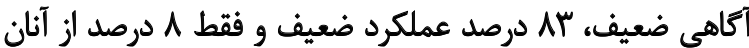

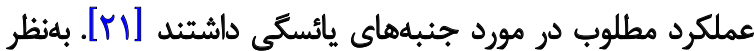

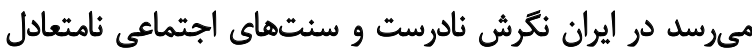

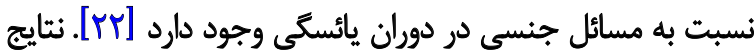

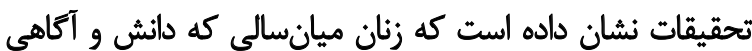

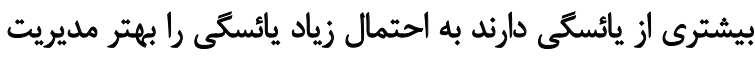

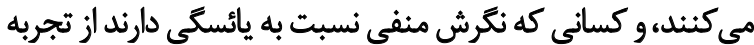

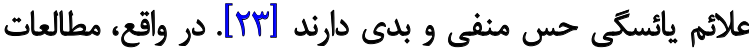

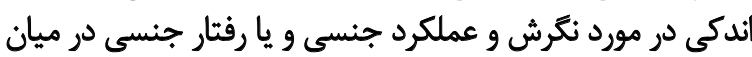

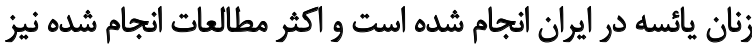

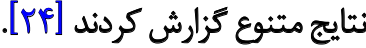

كليه نظامها و يستمهاي بهداشتى در جهان و همجنين نظامهاى

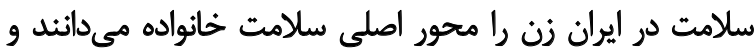

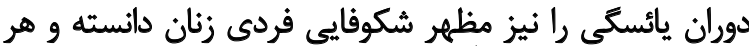

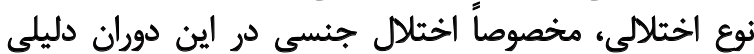

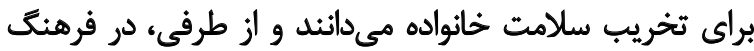

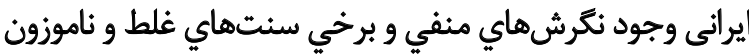

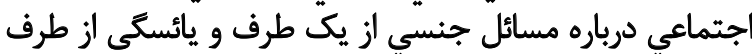

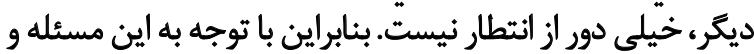

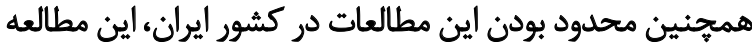

و هُرْشكان در ايجاد نكَرش مثبت زنان نسبت به يائسكى است

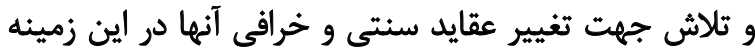

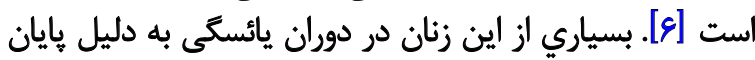

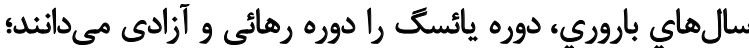

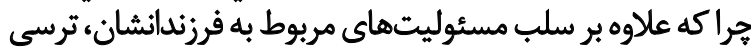

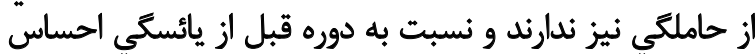

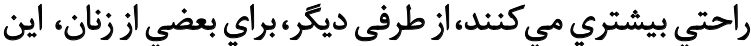

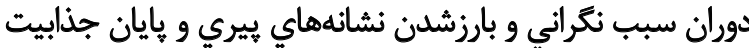
هايشان مىباشد [V] تغييرات بيولوزيك و هورمونى مرتبط با يائسكى در سرتاسر

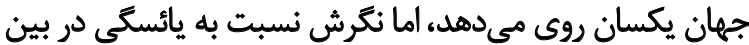

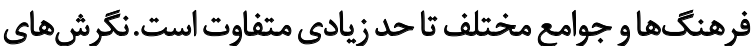

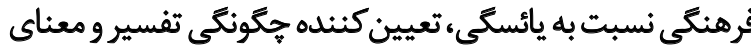

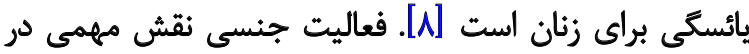

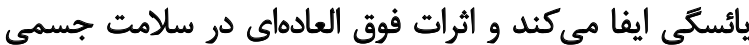

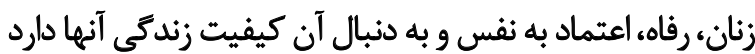

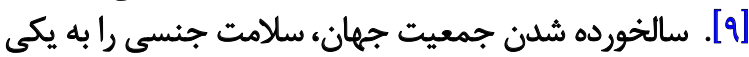

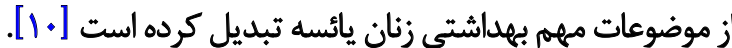
اكرجه عملكرد جنسى در دوران يائسكى تا حد زيادى تحت

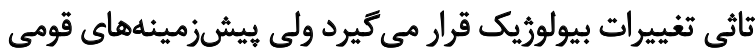

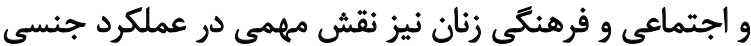

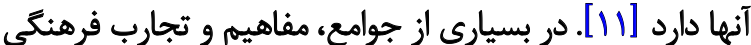

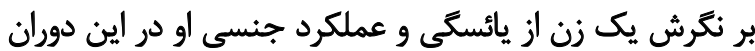

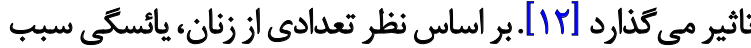

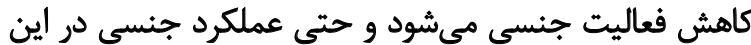

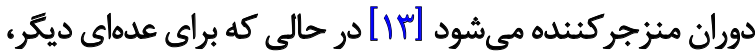

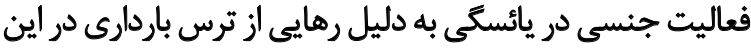

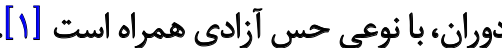
در تحقيق ديكر در نيجريه معلوم شد كه به طور سنتى، اين

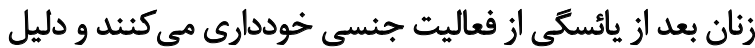

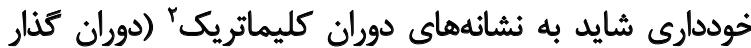

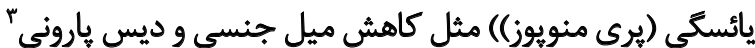

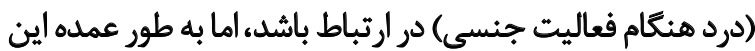

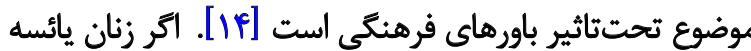

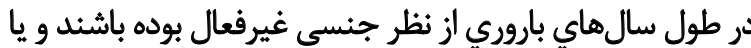

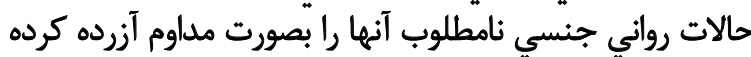

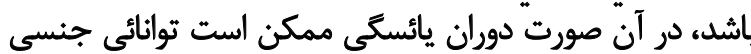

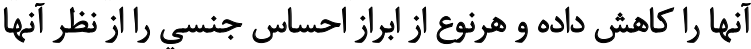

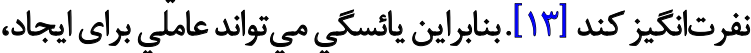

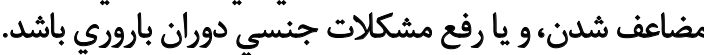


يائسكى نيوكارتن هو برسشنامه عملكرد جنسى زنان (FSFI) بود.

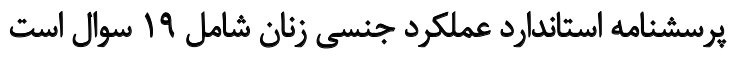

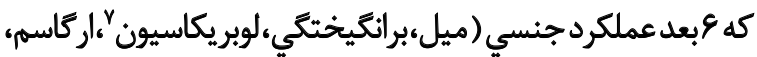

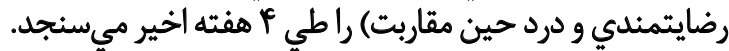

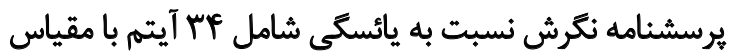

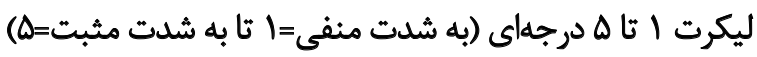

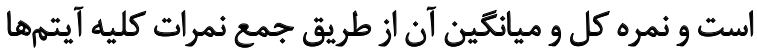

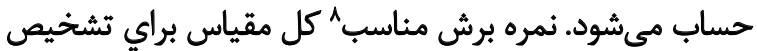

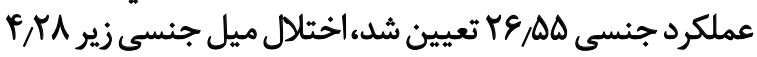

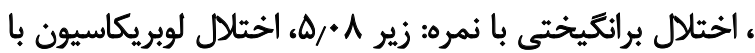

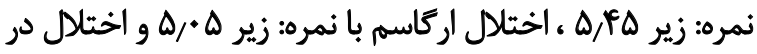

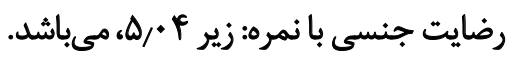

يرسشنامه نيوكارتن كه بهدنبال بررسى نكرش و احساسات

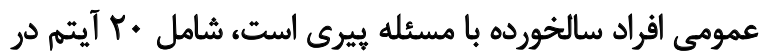

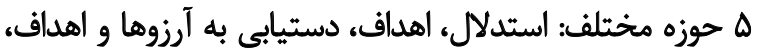

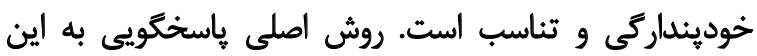

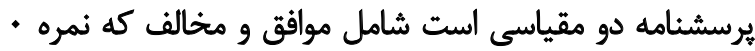

5. Neugarten

6. The Female Sexual Function Index (FSFI)

7. lubrication

8. Cut-off point
با هدف "بررسي رابطه بين عملكرد جنسي و نكرش نسبت به

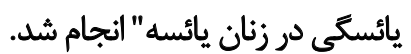
ووش مطإله

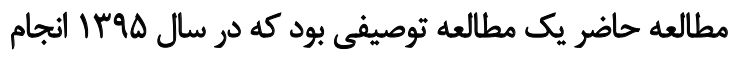

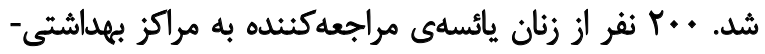

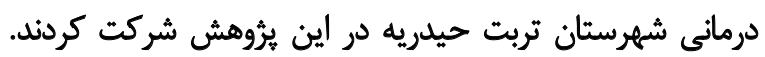

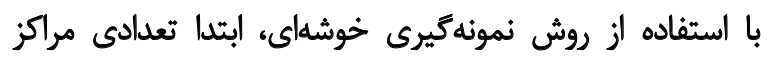

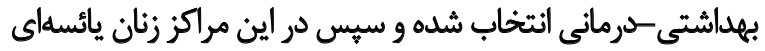

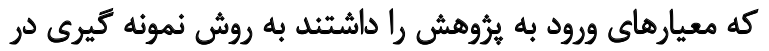

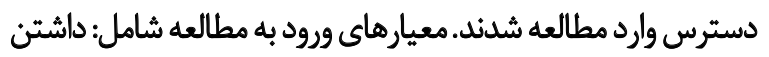

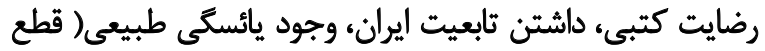

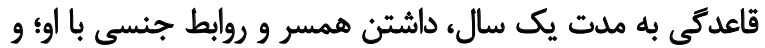

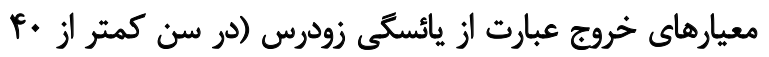

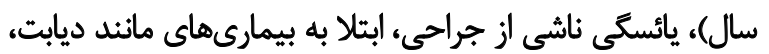

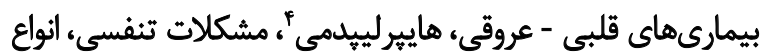

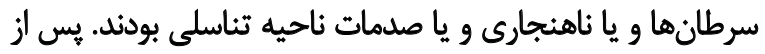

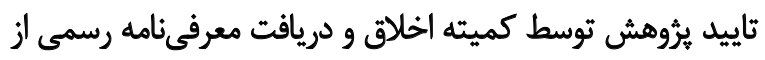

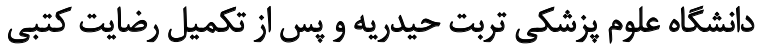

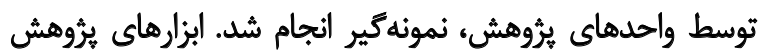

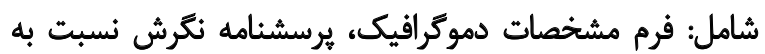

4. Hyperlipidemia

جدول ا.فراوانى حيطه هاى عملكرد جنسى زنان يائسه

\begin{tabular}{|c|c|c|}
\hline \multicolumn{2}{|c|}{ تعداد (درصد) } & \multirow{2}{*}{ حيطه عملكرد جنسي. } \\
\hline نامطلوب & مطلوب & \\
\hline $\operatorname{Mr}(\Delta / 91)$ & $\operatorname{IV}(\Delta / \Lambda)$ & تمايل \\
\hline$M \cdot(q \cdot)$ & $r \cdot(1 \cdot)$ & برانكيختكىى \\
\hline$W \in\left(9 Y^{w}\right)$ & If $(\mathrm{V})$ & لففزْندكى \\
\hline $1 \varepsilon_{\Delta}\left(\Lambda_{*}\right)$ & $r \cdot(r \cdot)$ & اركاسم \\
\hline$I f \Delta(\Delta / N)$ & $\Delta \Delta(\Delta / T V)$ & رضايت \\
\hline
\end{tabular}

$\stackrel{2}{2}$

جدول r. ارتباط بين عملكرد جنسي يا نكرش نسبت به يائسكى در زنان يائسه"

\begin{tabular}{|c|c|c|c|c|}
\hline \multirow{3}{*}{$\mathbf{P}$} & \multirow{3}{*}{ كل - - اكل } & \multicolumn{2}{|c|}{ تعداد (درصد) } & \multirow{3}{*}{ ملكرد جنسى } \\
\hline & & \multicolumn{2}{|c|}{ نكرش نسبت به يائسكى } & \\
\hline & & هُبت & منفي & \\
\hline \multirow{3}{*}{$.1 . \mathrm{V}$} & $\Delta \Delta(\Delta / T V)$ & $\operatorname{IV}(\Phi / \Lambda)$ & H人(19) & مطلوب \\
\hline & $I F \Delta(\Delta / M T)$ & $\Delta f(M)$ & 9) (ब/eब) & ن مامطلوب \\
\hline & $r \cdots(1+\cdots)$ & $n(\Delta / r \Delta)$ & Irq (A/AT) & 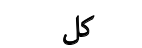 \\
\hline
\end{tabular}

L "نتايج آزمون كاى اسكوئر" 


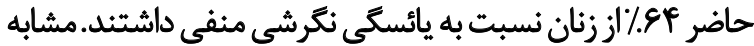

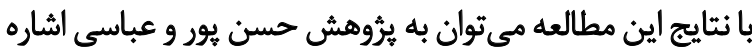

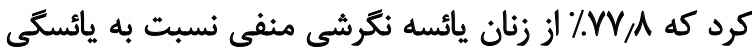

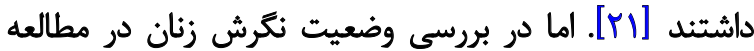

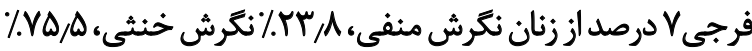

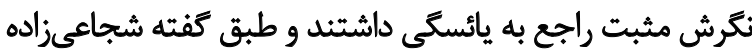

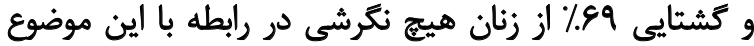

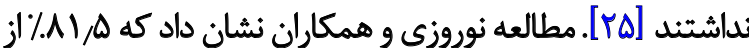

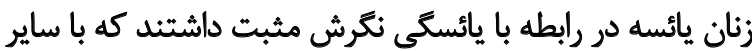

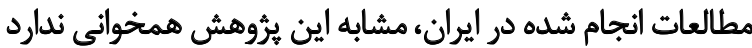

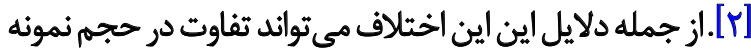

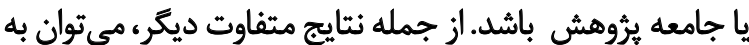

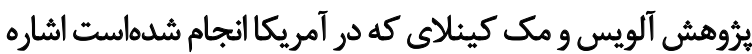

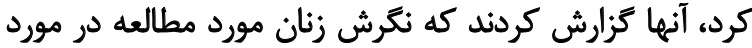

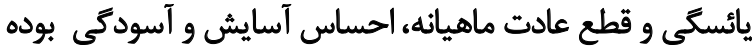

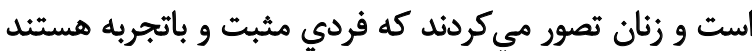

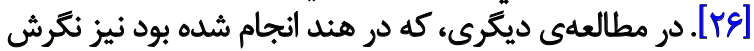

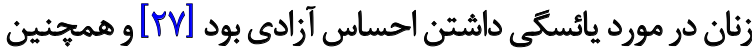

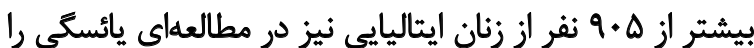

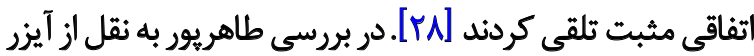

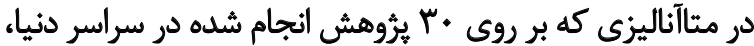

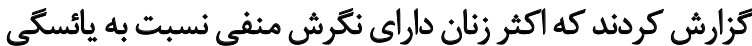

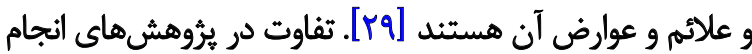

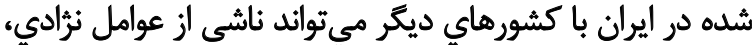

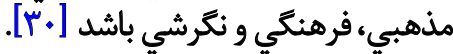
در رابطه با ثاثير عملكرد جنسى بر نتخرش نسبت به يائسكى دئى

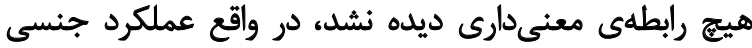

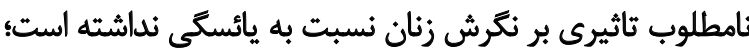

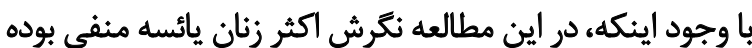

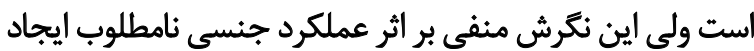

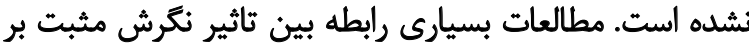

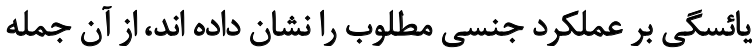

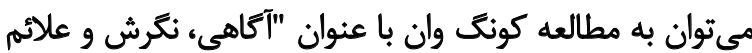

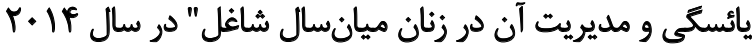

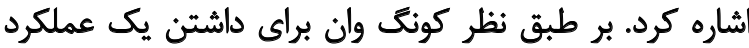

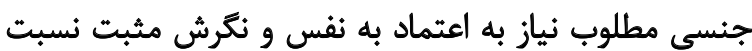

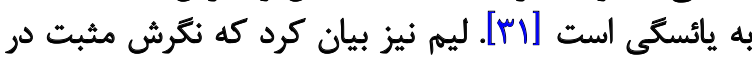

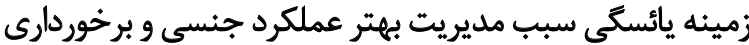

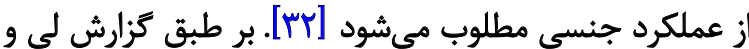

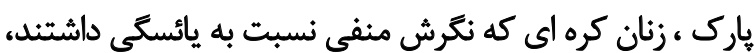

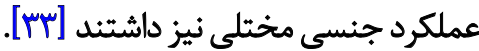
از جمله تحقيقاتى كه در ايران در اين زمينه انجام شده است،

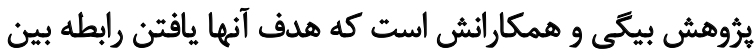
عملكرد جنسى و نكرش نسبت به يائسكى بود، نتايج نشان النيان

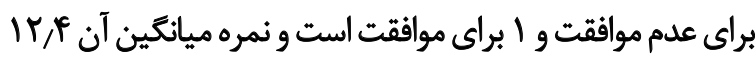

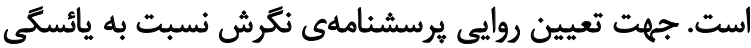

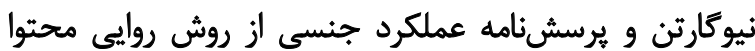

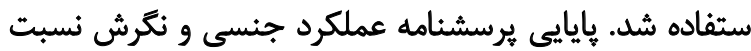

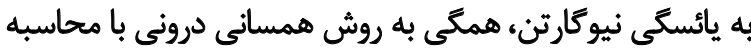

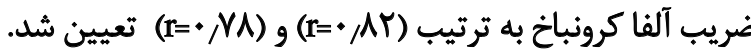

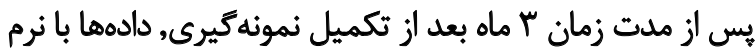

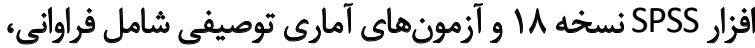

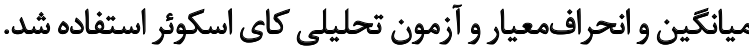

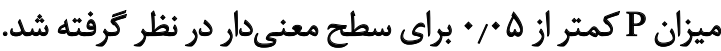

ياثتهها

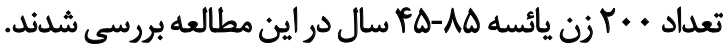

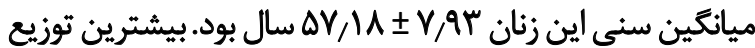

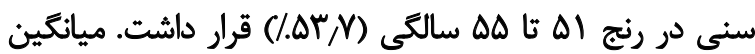

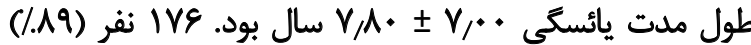

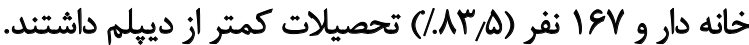

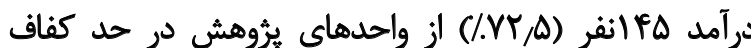

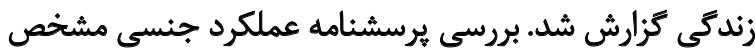

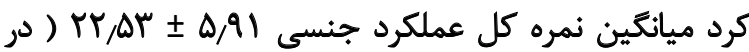

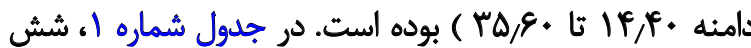

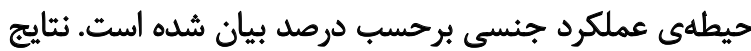

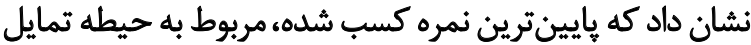

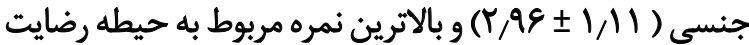

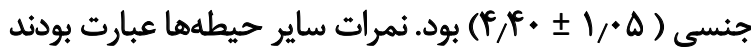

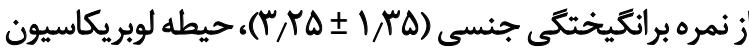

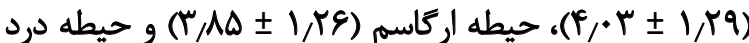
د (F)

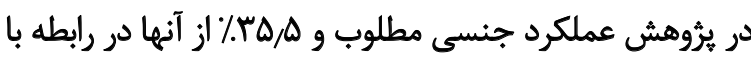
يائسگى نتخرشى مثبت داشتيند.

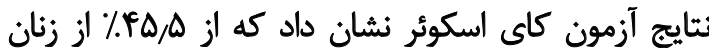

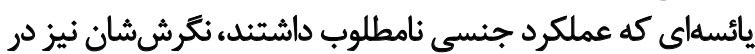

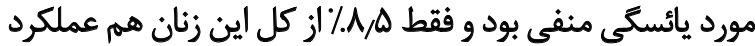

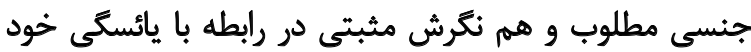

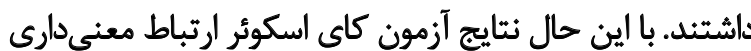

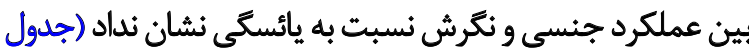

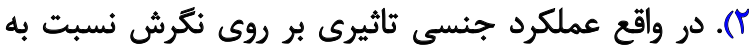

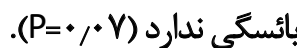

بحث مطالعه حاضر نشان داد بين عملكرد جنسى و نكرش نسبت

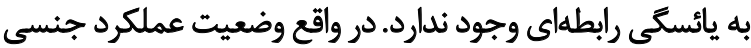

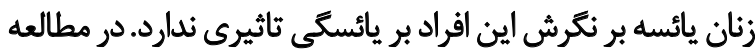




\section{نتيجهة تيرى نهايى}

با وجود اينكه ارتباطى بين عملكرد جنسى و نكرش نسبت

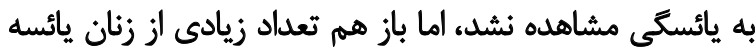

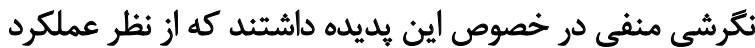

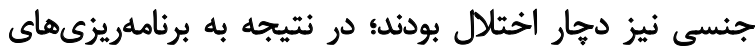

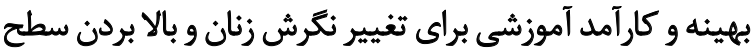
سلامت و كيفيت زندگى ايشان نياز است.

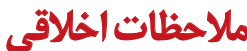

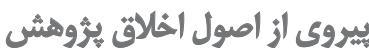

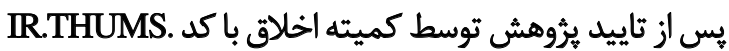

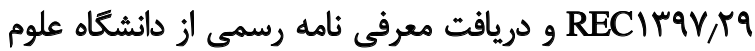

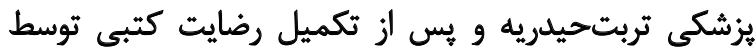

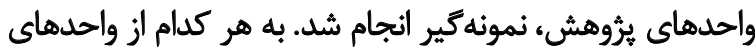

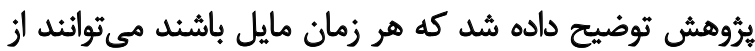

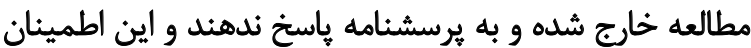

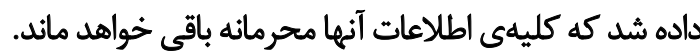

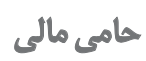

IR.THUMS. مطالعه حاضر حاصل طرح تحقيقاتى با كد

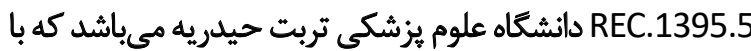

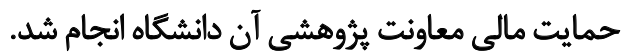

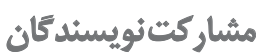

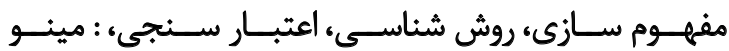

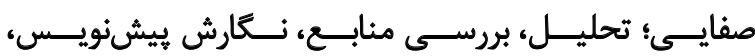

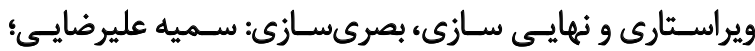

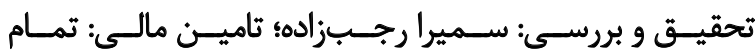
نويسـندان

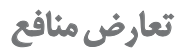

بنابر اظهار نويسندكان، اين مقاله تعارض منافع ندارد.

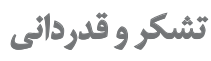

بدينوسيله از همكارى و مساعدت معاونت محترم يروهشى

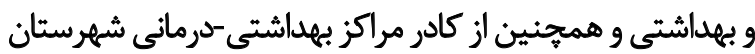

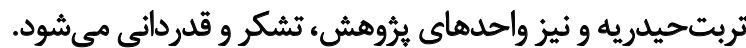

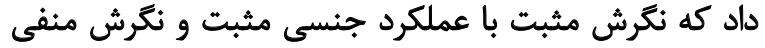

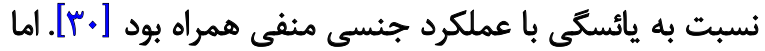

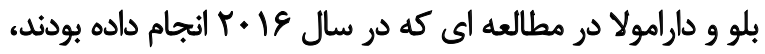

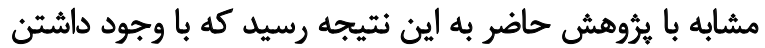

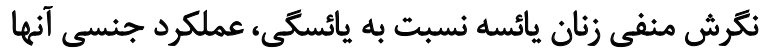

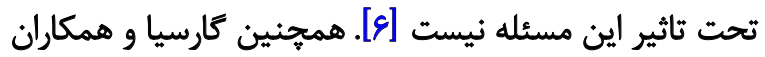

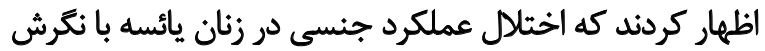

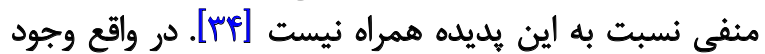

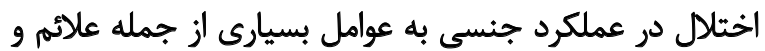

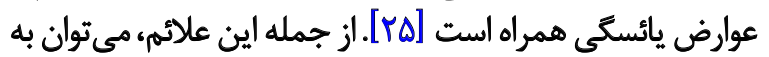

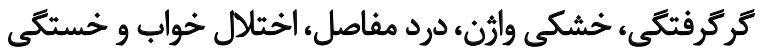

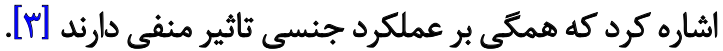

در ثروهش حاضر در تمام حيطههاى عملكرد جنسى اختلال

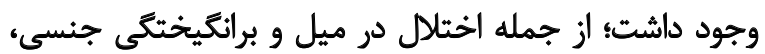

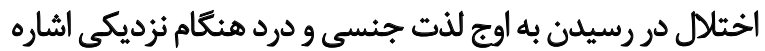

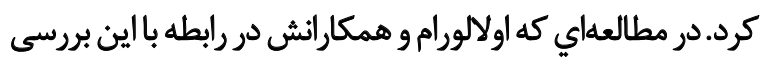

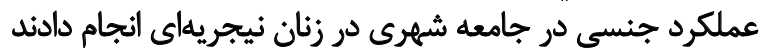

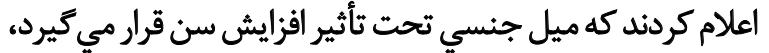

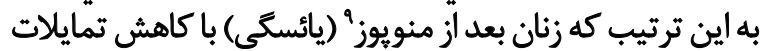

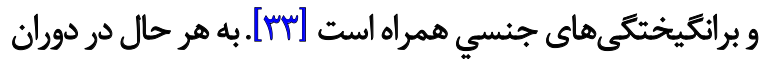

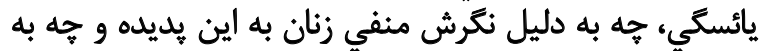

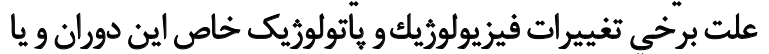

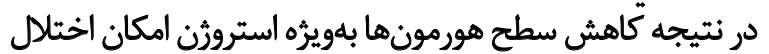

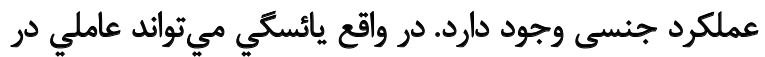

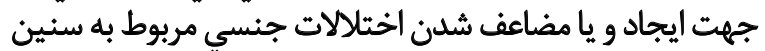

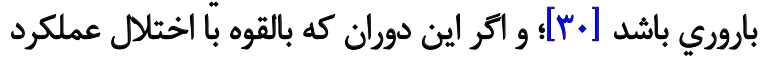

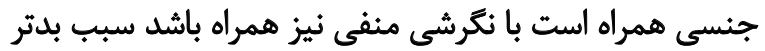

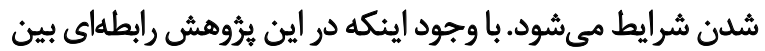

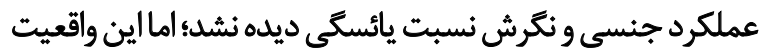

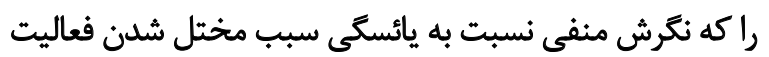

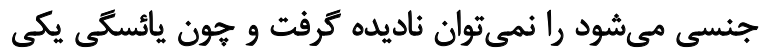

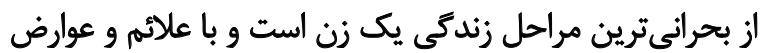

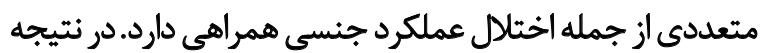

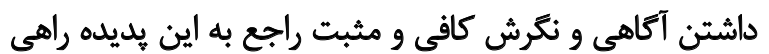

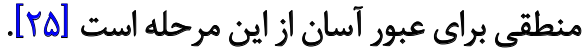

از جمله محدوديتهاى اين برؤوهش، دامنهى محدود نمونهما

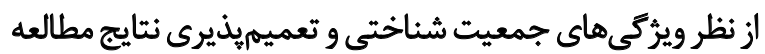

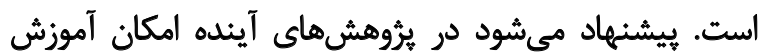

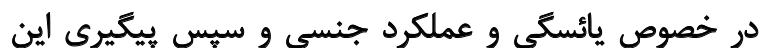
آموزشها فراهم شود. 


\section{References}

[1] Ande AB, Omu OP, Ande OO, Olagbuji NB. Features and perceptions of menopausal women in Benin City, Nigeria. Annals of African Medicine, 2011; 10(4):300-4. [DOI:10.4103/15963519.87048] [PMID]

[2] Noroozi E, Kasiri Dolatabadi N, Eslami AA, Hasanzadeh A, Davari S. Knowledge and Attitude of 40-45 Year Old Women Toward Menopause. Journal of Health Research System. 2012; 7(4):460-8.

[3] Dennerstein L Dudley E, Hopper J, Guthrie J, Burger H. A prospective population-based study of menopausal symptoms. Obstet Gynecol. 2000; 96:351-358. [DOI:10.1097/00006250200009000-00007]

[4] Ibraheem OM, Oyewole OE, Olaseha IO. Experiences and perceptions of menopause among women in Ibadan South East Local Government area, Nigeria. African Journal of Biomedical Research. 2015; 18(2):81-94.

[5] Palank CL. Determinants of health primitives' behavior. The Nursing Clinics of North America. 1998; 26(4):815-833.

[6] Bello F, Daramola O. Attitude to the menopause and sex amongst middle-aged women in a family medicine clinic in Ibadan, Nigeria. Obstetrics and Gynecology International. 2016; 1-5. [DOI:10.1155/2016/2031056] [PMID] [PMCID]

[7] Bahri N, Afiyat M, Aghamohamadiayan H, Delshad A, Bahri NA. The relationship between menopause and depression and anexiety. Iranian journal of obstetrics, gynecology and infertility. 2013; 16(43):14-20

[8] Hunter M, Odea I. An Evaluation Of A Health Education Intervention Mid -Aged Women :Five Year Follow-Up Of Effects Upon Knowledge ,Impact Of Menopause And Health. Patient Education and Counseling. 1999; 38(3):249-55. [DOI:10.1016/ S0738-3991(98)00143-8]

[9] Omidvar S, Bakouie F, Amiri FN. Sexual function among married menopausal women in Amol (Iran). Journal Midlife Health. 2011; 2:77-80. [DOI:10.4103/0976-7800.92534] [PMID] [PMCID]

[10] Moghassemi S, Ziaei S, Haidary Z. Effect of Tibolone on sexual function in healthy postmenopausal women.Journal of Gorgan University of Medical Sciences. 2010; 11(4):21-8.

[11] Avis NE, Zhao X, Johannes CB, Ory M, Brockwell S, Greendale GA. Correlates of sexual function among multi-ethnic middle-aged women: Results from the Study of Women's Health Across the Nation (SWAN). Menopause. 2005; 12:38598. [DOI:10.1097/01.GME.0000151656.92317.A9] [PMID]

[12] Dennerstein L, Alexander JL, Kotz K. The menopause and sexual functioning: a review of the population-based studies. Annual Review of Sex Research. 2003; 14:64-82.

[13] Ohadi B. Feelings and human sexual responses. $7^{\text {th }}$ Edition. Tehran: Naghsh Khorshid Publications; 2003.

[14] Dennerstein L, Smith AM, Morse C. Psychological wellbeing, mid-life and the menopause. Maturitas. 1994; 20(1):1-1. [DOI:10.1016/0378-5122(94)90095-7]

[15] Rotem M, Kushnir T, Levine R, Ehrenfeld M. A psycho - education program for improving women 's attitudes and coping with menopause symptom. Journal of Obstetric, Gynecologic \&
Neonatal. 2005; 34(2):233-40. [DOI:10.1177/0884217504274417] [PMID]

[16] Nappi RE, Nijland EA. Women's perception of sexuality around the menopause: Outcomes of a European telephone survey. Eur European Journal of Obstetrics \& Gynecology and Reproductive Biology. 2008; 137(1):10-6. [DOI:10.1016/j. ejogrb.2006.10.036] [PMID]

[17] Dennerstein L, Lehert P, Burger H. The relative effects of hormones and relationship factors on oophorectomy. The Journal of Clinical Endocrinology and Metabolism. 2005; 90:3847-5383.

[18] Peeyananjarassri K, Liabsuetrakul T, Soonthornpun K, Choobun T, Manopsilp P. Sexual functioning in postmenopausal women not taking hormone therapy in the Gynecological and Menopause Clinic, Songklanagarind Hospital measured by Female Sexual Function Index questionnaire. Journal of the Medical Association of Thailand. 2008; 91(5):625-32.

[19] Huang AJ, Subak LL, Thom DH, Van Den Eeden SK, Ragins AI, Kuppermann M, et al. Sexual function and aging in racially and ethnically diverse women. Journal of the American Geriatrics Society. 2009; 57(8):1362-8. [DOI:10.1111/j.15325415.2009.02353.x] [PMID] [PMCID]

[20] Szwabo PA. Counseling about sexuality in the older person Clinics in Geriatric Medicine. 2003;19(3):595-604. [DOI:10.1016/ S0749-0690(02)00113-1]

[21] Hassan Zadeh GR, Yekkezare S, Vaziri S, Soroorirad B, Darki L. Knowledge of women about menopause in Qazvin. The Journal of Qazvin University of Medical Sciences. 2003; 7(8):21-5.

[22] Lim KY. The study of menopause-related quality of life and management of climacteric in a middle-aged female population in Korea. Public Health Weekly Reports. 2013; 6:609-13.

[23] Hashemi S, Ramezani Tehrani F, Simbar M ,Abedini M, Bahreinian $\mathrm{H}$, Gholami R. Evaluation of sexual attitude and sexual function in menopausal age; a population based cross-sectional study. Iranian Journal of Reproductive Medicine. 2013; 11(8): 631-6.

[24] Faraji R, Asgharnia M, Hoseinzadeh F, Daliheirati SF, Emadi A. Attitude and knowledge women about menopause and hormone replacement therapy. Journal of Nursing and Midwifery Sciences. 2015; 24(71):48-55.

[25] Avis NE, McKinlay SM. A longitudinal analysis of women's attitudes toward the menopause: Results from the Massachusetts Women's Health Study. Maturitas. 1991; 13(1):65-79. [DOI:10.1016/0378-5122(91)90286-Y]

[26] Punyahotra S, Dennerstein L, Lehert P. Menopausal experiences of Thai women. Part 1: Symptoms and their correlates. Maturitas. 1997; 26(1):1-7. [DOI:10.1016/S0378-5122(96)010584]

[27] Donati S, Cotichini R, Mosconi P, Satolli R, Colombo C, Liberati A, et al. Menopause: Knowledge, attitude and practice among Italian women. Maturitas. 2009; 63(3): 246-252 [DOI:10.1016/j.maturitas.2009.04.001] [PMID]

[28] Taherpour M , Sefidi F. The Effectiveness of Education on the Knowledge and Attitude Towards Menopause Symptoms and Complications in Postmenopausal Women. Journal of Advances in Medical and Biomedical Research. 2012; 21(84):92-101. 
[29] Beigi M, Javanmardi Z, Abdolahi M. Sexual dysfunction at ri and post menopause. Avicenna Journal of Clinical Medicine. 2008; 16(2):37-43.

[30] Kyung Kwak E, Soon Park H, Kang N. menopause knowledge, attitude, symptom and management among midlife employed women. Journal of Mathematical Modeling. 2014; 20:118-25. [DOI:10.6118/jmm.2014.20.3.118] [PMID] [PMCID]

[31] Park JS, Lee YE. Effects of integrated menopause management program for middle aged woman. Korean Journal of Women Health Nursing. 2011;17:10-20. [DOI:10.4069/kjwhn.2011.17.1.10]

[32] Gracia C, Freeman E, Sammel M, Lin H, Mogul M. Hormones and sexuality during transition to menopause. Hormones and Sexuality Journal. 2007; 19(4):831-40. [DOI:10.1097/01. AOG.0000258781.15142.0d] [PMID]

[33] Olaoloram FM, Lawoyin TO. Experience of menopausal symptoms by women in an urban community in Ibadan, Nigeria. Menopause. 2009; 16(4):822-30.

[34] Trudeau KJ, Ainscough JL, Trant M, Starker J, Cousineau TM. Identifying the educational needs of menopausal women: a feasibility study. Women's Health Issues. 2011; 21(2):145-52. 